Int. J. Odontostomat., 11(4):495-499, 2017.

\title{
Caracterización de la Función Masticatoria en Estudiantes Universitarios
}

\author{
Characterization of the Masticatory Function in University Students
}

\author{
María Paz Moya ${ }^{1,2} ;$ Katherine Marquardt ${ }^{1}$ \& Sergio Olate ${ }^{2,3,4}$
}

MOYA, M. P. ; MARQUARDT, K. \& OLATE, S. Caracterización de la función masticatoria en estudiantes universitarios. Int. J. Odontostomat., 11(4):495-499, 2017.

RESUMEN: La masticación es una función orofacial aprendida y de relevancia para el desarrollo armónico del sistema estomatognático y craneofacial. Métodos no instrumentales para la evaluación de la masticación y antecedentes de caracterización de la función masticatoria en Chile son escasos. El objetivo de este estudio es caracterizar la función masticatoria en adultos jóvenes, específicamente el patrón masticatorio más prevalente, número promedio de ciclos masticatorios, así como el tiempo masticatorio promedio, empleando un alimento sólido. Fueron evaluados 92 sujetos, estudiantes universitarios, con edades de entre 18 y 26 años, sin distinción de sexo, con dentición permanente completa o solo ausencia del tercer molar. Los sujetos evaluados presentan mayor prevalencia de patrón masticatorio alternado (62 $\%), 15,1$ ciclos masticatorios en promedio y un tiempo masticatorio promedio de 11,9 s. Nuestros resultados podrían ser considerados valores de referencia para la evaluación clínica de la masticación en Chile.

PALABRAS CLAVE: Masticación; Patrón Masticatorio; Estudiantes.

\section{INTRODUCCIÓN}

La masticación es una de las funciones esenciales del sistema estomatognático (Yeerken et al., 2017). Esta corresponde al acto de morder, triturar y masticar el alimento; siendo considerada un proceso fisiológico complejo, que envuelve actividades neuromusculares dependientes del desarrollo del complejo craneofacial, del sistema nervioso central y de la oclusión dentaria (Susanibar et al., 2014).

La masticación es una función orofacial aprendida y de relevancia para el desarrollo armónico del sistema estomatognático y craneofacial, pues cuando se realiza de forma alterada, promueve el dislocamiento de los cóndilos de la mandíbula, con contactos simultáneos de trabajo, balanceo y deslizamientos (Tessitore \& Cattoni, 2009). Además, la masticación es una función oral indispensable relacionada con la salud física, mental y social durante toda la vida (Fukushima-Nakayama et al., 2017).
La función masticatoria puede ser evaluada a través de mediciones objetivas, considerando la eficiencia masticatoria, fuerza de mordida y habilidad para mezclar el alimento, o subjetivamente considerando la percepción de la capacidad masticatoria del paciente (Shibuya et al., 2013). Desde el punto de vista de la motricidad orofacial, la evaluación de la masticación considera diversos aspectos: número de ciclos masticatorios, patrón masticatorio, tiempo masticatorio, entre otros (Susanibar et al.)

Los ciclos masticatorios corresponden al movimiento rítmico que se genera producto de la combinación de movimientos de apertura, cierre, lateralidad, protrusión y retrusión en el proceso de la masticación (Malca, 2016), donde las características oclusales son importantes. De acuerdo con Simões (1998), una de las condiciones de la estabilidad oclusal es la masticación bilateral con protrusión durante la incisión.

\footnotetext{
${ }^{1}$ Facultad de Ciencias de la Salud, Universidad Autónoma de Chile, Temuco, Chile.

${ }^{2}$ Programa Doctorado en Ciencias Morfológicas, Facultad de Medicina, Universidad de La Frontera, Temuco, Chile.

${ }^{3}$ División de Cirugía Oral y Maxilofacial, Facultad de Odontología, Universidad de La Frontera, Temuco, Chile.

${ }^{4}$ Centro de Excelencia en Estudios Morfológicos y Quirúrgicos (CEMyQ), Universidad de La Frontera, Temuco, Chile.
} 
El patrón masticatorio o lado de preferencia masticatoria considera el porcentaje de ciclos masticatorios derechos o izquierdos utilizando evaluaciones clínicas subjetivas (López et al., 2014), clasificándolo en bilateral alternado, bilateral simultáneo, unilateral preferencial o unilateral crónico (Genaro et al., 2009).

El tiempo masticatorio o duración de la masticación incluye el tiempo entre la incisión del alimento, trituración, humectación con saliva, hasta el momento en que el alimento se transforma en bocados más pequeños y se deglute (Kijak et al., 2017).

Diversas investigaciones científicas (Remijn et al., 2016; Laird et al., 2016) se refieren a la función masticatoria, sin embargo, este tipo de material no aborda características específicas respecto de la motricidad orofacial, sino que se enfoca en la anatomofisiología, electrofisiología y elementos como la influencia del sabor del alimento en los movimientos masticatorios (Okada \& Shiga, 2017).

Por lo anterior, este estudio tiene como objetivo caracterizar la función masticatoria en adultos jóvenes, específicamente el patrón masticatorio más prevalente, número de ciclos masticatorios promedio, así como el tiempo masticatorio promedio.

\section{MATERIAL Y MÉTODO}

Se diseñó un estudio de tipo observacional con corte transversal, para el análisis de 92 sujetos, sin distinción de sexo, con dentición permanente completa o solo ausencia de tercer molar. La muestra fue seleccionada por conveniencia, previa firma de consentimiento informado, de acuerdo con los lineamientos éticos establecidos para tales efectos. Los sujetos incluidos fueron estudiantes universitarios con edades de entre 18 y 26 años. Se excluyeron los sujetos que presentaran tratamiento fonoaudiológico u ortodóncico previo, cirugías de cabeza y cuello o la presencia de algún síndrome o malformación craneofacial.

El presente estudio fue aprobado por el Comité de Ética de la Universidad Autónoma de Chile, con el No09-15.

Protocolo de evaluación masticatoria. El procedimiento de evaluación clínica de la masticación fue realizado por un evaluador previamente calibrado, siguiendo los lineamientos establecidos por Genaro et al. en el Examen Miofuncional Orofacial - MBGR (Fig. 1). Consta de la grabación de tres actos masticatorios para cada sujeto evaluado, empleando una porción de galleta tipo oblea, marca McKay Alteza con sabor vainilla, de tres centímetros de longitud.

Masticación: [ ] Sumar todos los puntos (mejor resultado $=0$ y peor $=10$ )

Si está alterada, es de origen [ ] funcional [] anatómica [] DATM [ ] otro

Masticación Habitual [ ] (Utilizar siempre el mismo alimento)

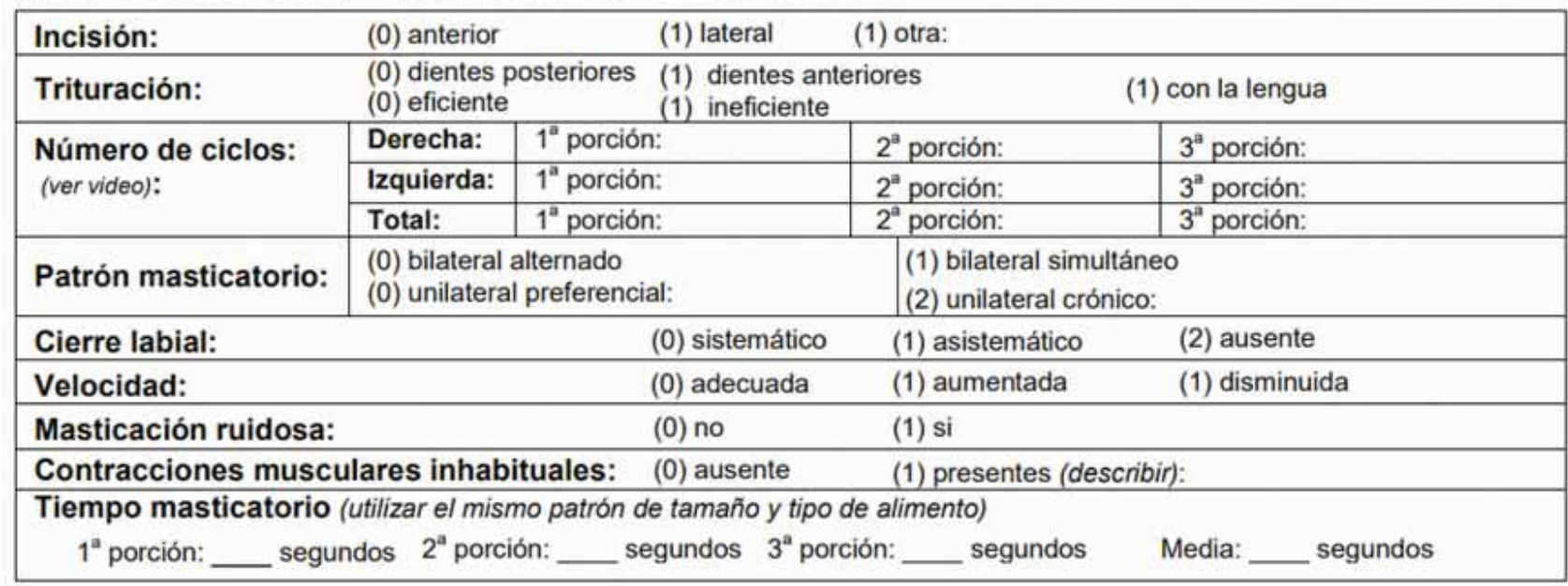

Observaciones:

Fig. 1. Evaluación de la función masticatoria (Genaro et al., 2009). 
Para la grabación del acto masticatorio se utilizó una cámara SONY Handycam HDR-CX190, ubicada frente al rostro del sujeto a un metro de distancia. El ambiente contaba con adecuadas condiciones de luz, sin distractores visuales y auditivos que puedan interferir en el proceso de evaluación.

Análisis Estadístico. Posterior a la evaluación de todos los sujetos, las grabaciones obtenidas fueron analizadas en términos de: duración promedio de masticación, número promedio de ciclos masticatorios e identificación del patrón masticatorio para cada sujeto de la muestra. Estos datos fueron tabulados en planilla Excel específica y procesados a través de Estadística descriptiva mediante el programa IBM SPSS Statistics versión 23. Se consideraron valores de frecuencia para variables categóricas, así como media y Desviación Stándar (DS) para variables continuas.

\section{RESULTADOS}

El estudio se desarrolló siguiendo el protocolo antes mencionado. Se observó que el patrón masticatorio en $62 \%$ de los sujetos evaluados corresponde al tipo alternado, 27,2 \% unilateral preferencial, 8,7 \% unilateral crónico y $2,1 \%$ a bilateral simultáneo (Tabla I).

Tabla I. Frecuencia de patrón masticatorio en estudiantes universitarios.

\begin{tabular}{lcc}
\hline Patrón Masticatorio & Frecuencia & $\%$ \\
\hline Bilateral Alternado & 57 & 62,0 \\
Unilateral Prefe rencial & 25 & 27,2 \\
Unilateral Crónico & 8 & 8,7 \\
Bilateral Simultáneo & 2 & 2,1 \\
Total & 92 & 100,0 \\
\hline
\end{tabular}

En relación a la cantidad promedio de los ciclos masticatorios, se obtuvo un valor de 15,1 ciclos (con un rango entre 7,6 y 24,0 ciclos). El tiempo promedio de la masticación se registró en 11,9 segundos, con un mínimo de 6,0 y un máximo de 20,6 segundos, para cada porción de alimento testado (Tabla II).

Tabla II. Promedio número de ciclos y tiempo, en estudiantes universitarios.

\begin{tabular}{lcccc}
\hline Promedio & Mínimo & Máximo & Media & D.S. \\
\hline Número de ciclos & 7,6 & 24,0 & 15,1 & 4,1 \\
Tiempo (s) & 6,0 & 20,6 & 11,9 & 3,1 \\
\hline
\end{tabular}

\section{DISCUSIÓN}

El presente estudio revela alta prevalencia de patrón masticatorio bilateral alternado en una población de adultos jóvenes, con una edad media de 22 años, que residen en la región de la Araucanía, Chile. De acuerdo con la Biblioteca del Congreso Nacional de Chile (2017), las principales actividades económicas de esta zona geográfica se vinculan a la agricultura, por lo que nuestros resultados pueden tener relación con las características de la dieta habitualmente ingerida. En ese sentido, diversos estudios refieren alta prevalencia de masticación bilateral alternada en poblaciones silvícolas, debido al consumo de alimentos duros y secos que proporcionan estimulación funcional adecuada para el desarrollo de los arcos dentarios y de todo el sistema estomatognático (Beyron, 1964; Van der Laan, 1998).

Monteiro et al. (2005) realizaron un estudio en 30 individuos con dispepsia funcional de ambos sexos, con un rango de edad entre 18 y 65 años, para determinar el lado de preferencia masticatoria de los participantes, obteniendo un patrón masticatorio predominante unilateral en el $46,7 \%$ de la muestra, seguido de masticación alternada (20\%) y bilateral simultánea $(23,3 \%)$. La investigación de Monteiro et al., sin embargo, tiene un sesgo importante toda vez que comparaciones entre grupos jóvenes de 18 años y adultos de 60 años pueden presentar diferencias relevantes. Nuestra investigación estudia sujetos adultos jóvenes a fin de obtener homogeneidad en la muestra demostrando un patrón bilateral alternado como el más prevalente $(62,0 \%)$, lo cual puede estar influenciado por la edad de los sujetos y las condiciones de oclusión.

Lima et al. (2006) identificaron el patrón masticatorio en niños entre 5 y 7 años de edad, siendo el de tipo alternado (38,5\%) el más prevalente. Asimismo, la edad y condición dentaria pueden influenciar en este aspecto ya que sujetos de entre 5 y 7 años presentan dentición mixta de primera fase con recambio dentario activo, lo que va a ser un factor relevante en el patrón de masticación.

Gomes et al. (2010) efectuaron un estudio conformado por 78 sujetos, 39 mujeres y 39 hombres, con una edad media de 23,5, determinando el tipo de masticación por medio de un kinesiógrafo (KNG), para registrar el movimiento mandibular durante la masticación de un material artificial de base de cau- 
cho, durante 15 ciclos de masticación. La mayor parte de la muestra registró un patrón alternado $(62,8 \%)$, seguido de un patrón unilateral preferencial $(37,2 \%)$; estos resultados son comparables a los que presenta nuestra investigación, considerando que el patrón alternado fue el más frecuente $(62,0 \%)$ seguido del patrón unilateral preferencial $(27,2 \%)$, de forma que podemos especular que en este grupo de edad, frecuentemente se observa un patrón normal de masticación.

Malca (2016), en 11 sujetos de ambos sexos con edades entre 12 y 29 años identificó el patrón masticatorio a través del protocolo MBGR. Para la evaluación fue utilizado un alimento sólido y el procedimiento fue registrado en un video para posteriormente realizar un análisis, obteniendo como resultado una mayor prevalencia del patrón unilateral preferencial $(54,54$ $\%)$ seguida del patrón alternado $(45,46 \%)$. Si bien el método empleado con nuestra investigación es semejante, la consistencia del producto masticable es muy diferente, de forma que el tipo de producto podría influenciar en estos resultados.

Gómez et al. (1998) denotan en su estudio variaciones de la duración promedio del ciclo masticatorio, al considerar diferentes alimentos. De Lucena et al. (2014) obtuvieron conclusiones semejantes, pues la duración promedio de ciclos masticatorios osciló entre 51 y 148 segundos, dependiendo del alimento testado.

Con estos antecedentes es evidente que el tiempo empleado en la función masticatoria dependerá de la consistencia del alimento, por lo que resultaría necesario contar con un protocolo de evaluación específico para la función masticatoria, estandarizando la cantidad y el tipo de alimento a utilizar para tales efectos. Al respecto, nuestro estudio ha considerado para la determinación de este parámetro, únicamente la masticación de un alimento sólido (trozo de galleta oblea), obteniendo un tiempo promedio de 11,9 segundos, valor que debe analizarse teniendo en cuenta que los alimentos más consistentes modifican el patrón de movimientos mandibulares, provocando el aumento de la extensión de los movimientos masticatorios y, por consiguiente, el aumento del tiempo total de masticación (Whitaker et al., 2009).

CONCLUSIONES. El patrón de masticación que se presenta con mayor prevalencia en los sujetos adultos jóvenes es de tipo alternado, permitiendo un equilibrado funcionamiento del sistema estomatognático.
Para la consistencia alimentaria testada se obtuvo 15,1 ciclos masticatorios promedio y un tiempo promedio de 11,9 segundos.

Es importante señalar que estos valores, al ser identificados en un grupo de sujetos con dentición permanente y sin patologías de base, podrían ser considerados valores de normalidad para su comparación en la evaluación clínica de la masticación.

AGRADECIMIENTOS. Los autores María Paz Moya y Katherine Marquardt agradecen a la Universidad Autónoma de Chile por financiar este estudio a través del Fondo de Investigación DIP99-16.

MOYA, M. P. ; MARQUARDT, K. \& OLATE, S. Characterization of the masticatory function in university students. Int. J. Odontostomat., 11(4):495-499, 2017.

ABSTRACT: Chewing is an orofacial function learned and relevant to the harmonic development of the stomatognathic system and craniofacial. Non-instrumental methods for the evaluation of chewing and registers of characterization of masticatory function in Chile are limited. The aim of this study is to characterize the masticatory function in young adults, specifically the most prevalent masticatory pattern, the mean masticatory cycles, as well as the mean masticatory time, using a solid food. Ninety-two subjects were evaluated, university students, with ages between 18 and 26 years, without distinction of sex, with complete permanent dentition or only absence of the third molar. The subjects evaluated showed a higher prevalence of alternate masticatory pattern (62\%), 15.1 masticatory cycles and masticatory time of $11.9 \mathrm{~s}$ on mean. Our results can be considered as reference values for the clinical evaluation of mastication in Chile.

Students.

KEY WORDS: Chewing; Masticatory Pattern;

\section{REFERENCIAS BIBLIOGRÁFICAS}

Beyron, H. L. Oclussal relations and mastication in Australian aborigines. Acta Odontol Scand., 22: 597-678, 1964.

Biblioteca del Congreso Nacional de Chile. Región de la Araucanía, 2017. Disponible en: https://www.bcn.cl/siit/ nuestropais/region9

De Lucena, C.; da Cunha, D.; de Oliveira, J. \& da Silva, H. Caracterização da mastigação segundo tempo, predominância de lateralidade e número de ciclos mastigatórios em adultos jovens. Rev. Disturb. Comun., 26(2):204-315, 2014. 
Fukushima-Nakayama, Y.; Ono, T.; Hayashi, M.; Inoue, M.; Wake, H.; Ono, T. \& Nakashima, T. Reduced Mastication Impairs Memory Function. J. Dent. Res., 96(9):1058-106, 2017.

Genaro, K. F.; Berretin-Félix, G.; Rehder, M. I. B. C. \& Marchesan, I. Q. Avaliação miofuncional orofacial - Protocolo MBGR. Rev. CEFAC, 11(2):237-55, 2009.

Gomes, S. G. F.; Custodio, W.; Jufer, J. S. M.; Cury, A. A. D. \& Garcia, R. C. M. R. Correlation of mastication and masticatory movements and effect of chewing side preference. Braz. Dent. J., 21(4):352-5, 2010.

Gómez, C. P. A.; Mesa, L. M. E. \& Vargas, I. D. J. Diseño y análisis de un sistema para medir los movimientos mandibulares. Rev. CES Odontol., 11(1):24-34, 1998.

Kijak, E.; Margielewicz, J.; Lietz-Kijak, D.; WilemskaKucharzewska, K.; Kucharzewski, M. \& S'liwin'ski, Z. Model identification of stomatognathic muscle system activity during mastication. Exp. Ther. Med., 13(1):13545, 2017.

Laird, M. F.; Vogel, E. R. \& Pontzer, H. Chewing efficiency and occlusal functional morphology in modern humans. J. Hum. Evol., 93:1-11, 2016.

Lima, R. M. F.; Freire, O. C. B.; Filho, J. L. N.; Stampford, S.; da Cunha, D. A. \& da Silva, H. J. Padrão mastigatório em crianças de 5 a 7 anos: Suas relações com crescimento craniofacial e hábitos alimentares. Rev. CEFAC, 8(2):20515, 2006.

López, O. P.; López, L. M.; Osorio, A. \& Restrepo, F. Lado de preferencia masticatoria en niños con fisura palatina: concordancia de tres métodos. Rev. Fac. Odontol., 26(1):89105, 2014.

Malca, L. Características funcionales orofaciales en pacientes jóvenes con mordida abierta interior. Tesis de Magíster en Fonoaudiología con mención en Motricidad Orofacial, Voz y Tartamudez. Lima, Pontificia Universidad Católica del Perú, 2016.

Monteiro, M.P.; Carneiro, F. P.; Felipe, N. A. P. \& Motta, A. R. Mastigação e dispepsia funcional: um novo campo de atuação. Rev. CEFAC, 7(3):340-7, 2005.

Okada, Y. \& Shiga, H. Effect of the bitterness of food on muscular activity and masticatory movement. J. Prosthodont. Res., 61(4):426-31, 2017.

Remijn, L.; Groen, B. E.; Speyer, R.; van Limbeek, J. \& Nijhuis-van der Sanden, M. W. Reproducibility of 3D kinematics and surface electromyography measurements of mastication. Physiol. Behav., 155:112-22, 2016.

Shibuya, Y.; Ishida, S.; Hasegawa, T.; Kobayashi, M.; Nibu, K., \& Komori, T. Evaluating the masticatory function after mandibulectomy with colour-changing chewing gum. J Oral Rehabil., 40(7):484-90, 2013.

Simões, W. A. Visão do crescimento mandibular e maxilar. J. Bras. Ortodon. Ortop. Facial., 3(15):9-18, 1998.

Susanibar, F.; Marchesan, I.; Parra, D. \& Dioses, A. Tratado de Evaluación de Motricidad Orofacial. Madrid, EOS, 2014.

Tessitore, A. \& Cattoni, D. Diagnóstico das Alterações de Respiração, Mastigação e Deglutição. En: Fernandez, F. D.; Mendes, B. C. \& Navas, A. L. (Eds). Tratado de fonoaudiologia. São Paulo, Roca, 2009.

Van der Laan, T. Função mastigatória em índios lanomami. Tese Mestrado em Ciências. Piracicaba, Universidade Estadual de Campinas, 1998.

Whitaker, M. E.; Júnior, A. S. T. \& Genaro, K. F. Proposta de protocolo de avaliação clínica da função mastigatória. Rev. CEFAC, 11(3):311-23, 2009.

Yeerken, Y.; Otomaru, T.; Said, M.; Li, N. \& Taniguchi, H. Applicability of the CIELAB and CIEDE2000 Formulae for Detection of Colour Changes in Colour-Changeable Chewing Gum for Evaluating Masticatory Function. J. Clin. Diagn. Res., 11(4):ZC119-ZC23, 2017.

Dirección para Correspondencia:

Prof. Dr. Sergio Olate

División de Cirugía Oral y Maxilofacial

Universidad de La Frontera

Claro Solar 115, Of. 414-A

Temuco

CHILE

Email: sergio.olate@ufrontera.cl

Recibido : 24-09-2017

Aceptado: 17-10-2017 